\title{
Associations of Common Variants at APLN and Hypertension in Chinese Subjects with and without Diabetes
}

\author{
Rong Zhang, Jingyi Lu, Cheng Hu, Congrong Wang, Weihui Yu, Feng Jiang, Shanshan Tang, \\ Yuqian Bao, Kunsan Xiang, and Weiping Jia
}

\begin{abstract}
Department of Endocrinology and Metabolism, Shanghai Jiao Tong University Affiliated Sixth People's Hospital, Shanghai Diabetes Institute, Shanghai Key Laboratory of Diabetes Mellitus, Shanghai Clinical Center for Diabetes, 600 Yishan Road Shanghai, 200233, China
\end{abstract}

Correspondence should be addressed to Weiping Jia, wpjia@sjtu.edu.cn

Received 27 April 2012; Revised 13 November 2012; Accepted 13 November 2012

Academic Editor: P. Nilsson

Copyright ( $) 2012$ Rong Zhang et al. This is an open access article distributed under the Creative Commons Attribution License, which permits unrestricted use, distribution, and reproduction in any medium, provided the original work is properly cited.

\begin{abstract}
Background. Apelin, the endogenous ligand for the APJ receptor, has a potent hypotensive effect via a nitric oxide-dependent mechanism in vivo. The aim of the study was to investigate the association between the common variants of apelin gene (APLN) and hypertension, which was reported recently in a Chinese Han population with and without diabetes. Methods. Three single nucleotide polymorphisms (SNPs) on APLN were genotyped in 3156 diabetic patients and 3736 nondiabetic individuals. For non-diabetic subjects, 1779 were enrolled in stage 1 and 1757 were recruited for validation. A meta-analysis combining the two stages was carried out to obtain the overall effect. Results. In diabetic patients, no significant associations of the three SNPs with hypertension were observed. In contrast, we found that rs2235306 was associated with hypertension in non-diabetic males after adjusting for covariates $(\mathrm{OR}=1.19, P=0.039)$ while rs2235307 and rs3115759 displayed no evidence of association in both genders. One haplotype, $\mathrm{C}-\mathrm{C}-\mathrm{A}$, also showed an association with hypertension $(\mathrm{OR}=1.47, P=0.032)$ only in men. However, analysis in stage 2 and meta-analysis did not support these findings. Conclusions. We conclude that common variants on APLN are not associated with the prevalence of hypertension in the Chinese.
\end{abstract}

\section{Introduction}

Essential hypertension is a major risk factor for many common causes of morbidity and mortality including stroke, myocardial infarction, heart failure, and end-stage renal disease [1-3]. Approximately, $30-50 \%$ of blood pressure variation in the general population is determined by genetic factors $[4,5]$ and a variety of gene variants have been shown to be associated with essential hypertension $[6,7]$.

Apelin, the endogenous ligand for the APJ receptor (an orphan G-protein-coupled receptor), is a bioactive peptide that is expressed in a wide variety of tissues [8-11]. The apelin gene (APLN) in humans is located on chromosome Xq25-26.1, which encodes a 77-amino acid prepropeptide that is cleaved into isoforms of varying lengths [8-13]. During the recent years, there is a mounting evidence that apelin has pleiotropic effects on lipid and glucose metabolisms [14-16] and therefore is correlated with metabolic disorders including diabetes. On the other side, since the apelin/APJ system has high sequence homology to the angiotensin II/angiotensin receptor (AII/AT) system [17], it has gained much research interest regarding its role in blood pressure control. By binding to APJ, apelin exerts a potent hypotensive effect via a nitric oxide-dependent mechanism in vivo [12, $18,19]$. In accord with this, circulating apelin was reported to be decreased in patients with essential hypertension [20,21], suggesting an important role for the apelin/APJ system in human blood pressure regulation.

To date, two studies have reported the associations of common genetic variants of APLN with hypertension [22, 23]. However, genetic findings need replication. Thus, we sought to investigate the relationship between APLN tagging SNPs and hypertension in a community-based population free of diabetes, excluding the confounding effect of diabetes on hypertension. On the other hand, hypertension and type 2 diabetes frequently coexist. There is a compelling evidence 
that hypertension could significantly increase the risk of cardiovascular diseases in diabetic patients [24], indicating an urgent need for identifying hypertension susceptible genes in this subset of individuals [25]. Moreover, dysfunctional endothelium-dependent vasodilation and impaired nitric oxide bioavailability have been reported in diabetes [26-28], raising the possibility that apelin may interact with diabetes in the regulation of blood pressure. In this context, another aim of the current study was to examine the effect of APLN genetic variants on hypertension in patients with diabetes.

\section{Materials and Methods}

2.1. Subjects. Diabetic patients were selected from the inpatient database of the Shanghai Diabetes Institute. Diabetes was defined according to the 1999 WHO criteria (fasting plasma glucose $\geq 7.0 \mathrm{mmol} / \mathrm{L}$ and/or $2 \mathrm{~h}$ plasma glucose $\geq 11.1 \mathrm{mmol} / \mathrm{L})$. Nondiabetic subjects were enrolled from the Shanghai Diabetes Study [29], a community-based epidemiological survey for diabetes. Briefly, the Huayang and Caoyang communities, two middle-income communities in Shanghai, were selected for the survey. The target population included unrelated residents over 40 years of age who had been living in Shanghai for more than 10 years. Normal glucose regulation was confirmed by fasting plasma glucose $(<6.1 \mathrm{mmol} / \mathrm{L})$ and $2 \mathrm{~h}$ plasma glucose after oral glucose tolerance test $(<7.8 \mathrm{mmol} / \mathrm{L})$. All subjects were of the Han Chinese ancestry and resided in Shanghai or nearby regions. Individuals who suffered from secondary hypertension, cancer, severe disability or severe psychiatric disturbance were excluded. In the analyses restricted to non-diabetic individuals, we used a two-stage strategy. In stage 1 , a total of 1779 (728 men and 1051 women) subjects were recruited. In stage 2, we additionally enrolled 1757 (668 men and 1089 women) subjects to validate the findings from stage 1 , following the same inclusion and exclusion criteria as in stage 1 . As for diabetic patients, 3156 were finally recruited.

General anthropometric parameters, including height, weight, and blood pressure, were measured in all participants. BMI was calculated as weight $(\mathrm{kg}) /$ height $^{2}(\mathrm{~m})^{2}$. Blood pressure was measured twice with standard mercury sphygmomanometers in each participant while seated, after 5 minutes of rest and the average of two readings was taken. Essential hypertension was defined as blood pressure $\geq 140 / 90 \mathrm{~mm} \mathrm{Hg}$ and/or the current use of antihypertensive medications. While the information on smoking was not available in stage 1, we obtained this information using questionnaires in stage 2. The study protocol was approved by the Institutional Review Board of Shanghai Jiao Tong University Affiliated Sixth People's Hospital, Shanghai, China. All participants gave informed consent prior to the study.

2.2. Clinical Laboratory Tests. Blood samples were collected after an overnight fast. Serum lipid profiles, including total cholesterol, triglyceride, high-density lipoprotein cholesterol (HDL-C), and low-density lipoprotein cholesterol (LDL-C), were measured with a type 7600-020 automated analyzer (Hitachi, Tokyo, Japan).

2.3. SNP Selection and Genotyping. Genomic DNA was extracted from peripheral blood leukocytes using the standard phenol-chloroform method. Based on the database of the HapMap Chinese Hans in Beijing (CHB) Group, three SNPs (rs2235307, rs2235306, and rs3115759) that captured all variants with minor allele frequencies over 0.05 in the APLN region under the threshold of $r^{2}=0.8$ were selected in this study by Tagger incorporated in Haploview (version 4.1) [30]. rs2235307, rs2235306, and rs3115759 map to intron 1, intron 2, and exon 3 of APLN, respectively. The genotyping was performed by matrix-assisted laser desorption ionization time of flight mass spectroscopy (MassARRAY Compact Analyzer, Sequenom, San Diego, CA, USA). The genotyping success rates for rs2235307, rs2235306, and rs3115759 were $98.7 \%, 98.7 \%$, and $99.5 \%$, respectively. The average concordance rate based on 100 duplicate pairs was $99.3 \%$ for each SNP.

2.4. Statistical Analysis. As APLN is located on the $\mathrm{X}$ chromosome, the genotype-hypertension analysis was conducted in men and women separately. Allele, genotype, and haplotype frequencies for cases and controls were compared by using $\chi^{2}$-test. Logistic regression analysis after adjustment for age, BMI, and FPG (for non-diabetic subjects) or diabetes duration (for diabetic patients) was carried out to evaluate the effect of genotypes and alleles on the prevalence of hypertension. Odds ratios (ORs) with 95\% confidence interval (95\% CI) were presented. A pairwise linkage disequilibrium (LD) was estimated by calculating $\left|D^{\prime}\right|$ and $r^{2}$ using Haploview (version 4.1). The haplotype block structure was determined using confidence interval algorithm [31], and haplotype frequencies were estimated by the expectationMaximization algorithm [32] using Haploview (version 4.1). Haplotypes with a frequency $<0.05$ were excluded. All statistical analyses were performed by SAS (version 8.0; SAS Institute Inc., Cary, NC, USA) unless specified otherwise. A two-tailed $P$ value $<0.05$ was considered statistically significant. Combined ORs and 95\% CIs were calculated with Review Manager (version 5.0). Interstudy heterogeneity was estimated using the $\chi^{2}$-based Q statistic. Heterogeneity was considered statistically significant when $P<0.05 . I^{2}$ was also tested, which describes the percentage of total variation in point estimates attributable to genuine variation rather than sampling error. If heterogeneity existed, data were analyzed using a random effect model while in the absence of heterogeneity, a fixed effect model was used.

\section{Results}

Table 1 presents the characteristics of diabetic patients. The characteristics of non-diabetic subjects are shown in Supplemental Table 1 (See Supplementry Material available at doi:10.1155/2012/917496). All the three SNPs conformed to the Hardy-Weinberg equilibrium in both controls and total participants. 
TABLE 1: The characteristics of diabetic patients.

\begin{tabular}{|c|c|c|c|c|c|c|}
\hline \multirow{2}{*}{ Parameters } & \multicolumn{3}{|c|}{ Men } & \multicolumn{3}{|c|}{ Women } \\
\hline & $\mathrm{NT}(n=653)$ & $\mathrm{HT}(n=997)$ & $P$ & $\mathrm{NT}(n=457)$ & $\mathrm{HT}(n=1049)$ & $P$ \\
\hline Age (years) & $53.92 \pm 12.84$ & $61.62 \pm 12.30$ & $<0.001$ & $58.8 \pm 12.05$ & $65.24 \pm 10.43$ & $<0.001$ \\
\hline BMI & $23.30 \pm 3.37$ & $24.62 \pm 3.20$ & $<0.001$ & $23.04 \pm 3.34$ & $24.60 \pm 3.70$ & $<0.001$ \\
\hline Onset age (years) & $48.77 \pm 11.58$ & $53.30 \pm 11.49$ & $<0.001$ & $52.63 \pm 11.12$ & $56.02 \pm 11.09$ & $<0.001$ \\
\hline Diabetes duration (years) & $5.39 \pm 5.47$ & $7.37 \pm 6.74$ & $<0.001$ & $7.73 \pm 6.48$ & $9.15 \pm 7.22$ & 0.030 \\
\hline $\mathrm{SBP}(\mathrm{mm} \mathrm{Hg})$ & $119.95 \pm 9.67$ & $141.00 \pm 16.71$ & $<0.001$ & $120.79 \pm 9.82$ & $144.84 \pm 17.84$ & $<0.001$ \\
\hline $\mathrm{DBP}(\mathrm{mm} \mathrm{Hg})$ & $75.60 \pm 6.40$ & $84.99 \pm 9.86$ & $<0.001$ & $74.65 \pm 6.69$ & $83.11 \pm 9.84$ & $<0.001$ \\
\hline Total cholesterol (mmol/L) & $4.47 \pm 1.00$ & $4.70 \pm 1.21$ & $<0.001$ & $4.88 \pm 1.06$ & $5.01 \pm 1.20$ & 0.097 \\
\hline Triglyceride (mmol/L) & $1.79 \pm 2.04$ & $1.98 \pm 2.05$ & $<0.001$ & $1.67 \pm 1.41$ & $2.02 \pm 1.81$ & $<0.001$ \\
\hline $\mathrm{HDL}(\mathrm{mmol} / \mathrm{L})$ & $1.08 \pm 0.30$ & $1.10 \pm 0.33$ & 0.330 & $1.30 \pm 0.40$ & $1.26 \pm 0.73$ & $<0.001$ \\
\hline $\mathrm{LDL}(\mathrm{mmol} / \mathrm{L})$ & $2.89 \pm 0.91$ & $3.00 \pm 0.94$ & 0.016 & $3.06 \pm 0.88$ & $3.12 \pm 0.96$ & 0.299 \\
\hline
\end{tabular}

Continuous variables were means \pm SD. Categorical variables were numbers with percentages. NT: normotensive subjects. HT: hypertensive subjects. BMI: body mass index. SBP: systolic blood pressure. DBP: diastolic blood pressure. HDL: high-density lipoprotein cholesterol. LDL: low-density lipoprotein cholesterol.

TABLE 2: Analysis for the association of the three SNPs with hypertension in diabetic males.

\begin{tabular}{|c|c|c|c|c|c|c|}
\hline SNP & Allele & NT $(n=653)$ & $\mathrm{HT}(n=997)$ & OR $(95 \% \mathrm{CI})$ & $P$ & $P^{*}$ \\
\hline \multirow{2}{*}{ rs2235307 } & $\mathrm{C}$ & $520(80.37 \%)$ & $809(82.38 \%)$ & \multirow{2}{*}{$0.83(0.59-1.15)$} & \multirow{2}{*}{0.305} & \multirow{2}{*}{0.250} \\
\hline & $\mathrm{T}$ & $127(12.93 \%)$ & $173(17.62 \%)$ & & & \\
\hline \multirow{2}{*}{ rs2235306 } & $\mathrm{T}$ & $377(58.27 \%)$ & $538(54.73 \%)$ & \multirow{2}{*}{$1.17(0.90-1.50)$} & \multirow{2}{*}{0.159} & \multirow{2}{*}{0.239} \\
\hline & $\mathrm{C}$ & $270(41.73 \%)$ & $445(45.27 \%)$ & & & \\
\hline \multirow{2}{*}{ rs3115759 } & $\mathrm{A}$ & $442(67.69 \%)$ & $673(67.64 \%)$ & \multirow{2}{*}{$0.94(0.72-1.22)$} & \multirow{2}{*}{0.983} & \multirow{2}{*}{0.639} \\
\hline & G & $211(32.31 \%)$ & $322(32.36 \%)$ & & & \\
\hline
\end{tabular}

NT: normotensive subjects. HT: hypertensive subjects.

${ }^{*} P$ values were adjusted for age, body mass index and diabetes duration.

For diabetic patients, the allele and genotype distributions of the SNPs genotyped did not differ significantly between hypertensive and normotensive individuals in males (Table 2) and females (Table 3), respectively. Adjusting for covariates including age, BMI, and diabetes the duration, the logistic regression analysis did not reveal any significant associations (Tables 2 and 3), either.

Based on the $\left|D^{\prime}\right|$ and $r^{2}$ values, the three SNPs were located in one block (Figure 1). Four haplotypes, constructed by rs 2235307, rs 2235306 , and rs 3115759 , with a frequency of $>0.05$ were observed in our study samples (Table 4 ). None of them exhibited a significantly differed distribution between hypertensive and normotensive participants in the diabetes group.

Concerning non-diabetic participants (stage 1), we found that rs2235306, but not rs2235307 and rs3115759, was associated with the prevalence of hypertension only in men (Supplemental Tables 2 and 3) after adjusting for covariates $(\mathrm{OR}=1.19,95 \% \mathrm{CI}: 1.01-1.40, P=0.039)$, with the minor allele $(\mathrm{C})$ being more frequent in hypertensive subjects. Moreover, the C-C-A haplotype (rs2235307rs2235306-rs3115759) was related to with a higher risk of hypertension $(\mathrm{OR}=1.47,95 \% \mathrm{CI} 1.09-2.00, P=0.032)$ in men (Supplemental Table 4). No association of the other three haplotypes with hypertension was observed.
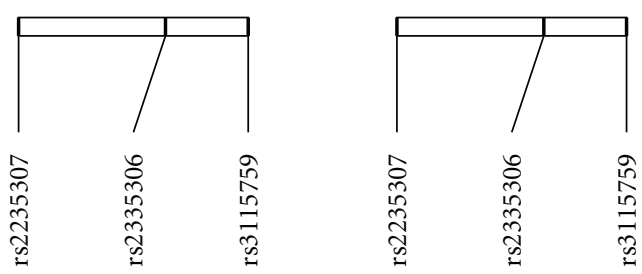

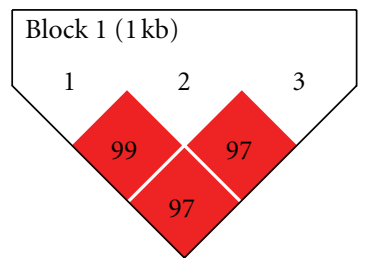

(a)

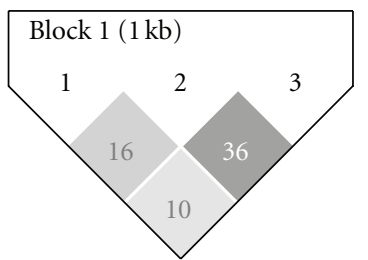

(b)
Figure 1: Pairwise linkage disequilibriums of SNPs genotyped in the APLN region. (a) Numbers represent $\left|D^{\prime}\right|$ expressed as a percentage. (b) Numbers represent $r^{2}$ expressed as a percentage.

Since the rs2235306 C allele and the associated haplotype C-C-A correlated with a higher hypertension risk in nondiabetic males, we genotyped the three SNPs in an additional group (stage $2, n=1757$ ) of non-diabetic subjects. In these samples, no evidence for the associations of rs2235306 
TABLE 3: Analysis for the association of the three SNPs with hypertension in diabetic females.

\begin{tabular}{|c|c|c|c|c|c|c|}
\hline SNP & Genotype & NT & $\mathrm{HT}$ & OR $(95 \% \mathrm{CI})$ & $P$ & $P^{*}$ \\
\hline \multirow{3}{*}{ rs2235307 } & $\mathrm{CC}$ & $288(63.6 \%)$ & $712(68.8 \%)$ & \multirow{3}{*}{$0.88(0.54-1.42)$} & \multirow{3}{*}{0.143} & \multirow{3}{*}{0.592} \\
\hline & CT & $150(33.1 \%)$ & $293(28.3 \%)$ & & & \\
\hline & TT & $15(3.3 \%)$ & $30(2.9 \%)$ & & & \\
\hline \multirow{3}{*}{ rs2235306 } & TT & $150(33.5 \%)$ & $318(30.7 \%)$ & \multirow{3}{*}{$1.19(0.80-1.76)$} & \multirow{3}{*}{0.562} & \multirow{3}{*}{0.390} \\
\hline & $\mathrm{TC}$ & $217(48.4 \%)$ & $525(50.6 \%)$ & & & \\
\hline & $\mathrm{CC}$ & $81(18.1 \%)$ & $194(18.7 \%)$ & & & \\
\hline \multirow{3}{*}{ rs3115759 } & AA & $207(45.3 \%)$ & $464(44.2 \%)$ & \multirow{3}{*}{$0.78(0.53-1.15)$} & \multirow{3}{*}{0.485} & \multirow{3}{*}{0.212} \\
\hline & AG & $193(42.2 \%)$ & $472(45.0 \%)$ & & & \\
\hline & GG & $57(12.5 \%)$ & $113(10.8 \%)$ & & & \\
\hline
\end{tabular}

NT: normotensive subjects. HT: hypertensive subjects.

* $P$ values were adjusted for age, body mass index, and diabetes duration.

TABLE 4: Analysis for the association of haplotypes with hypertension in diabetic patients stratified by gender.

\begin{tabular}{|c|c|c|c|c|c|c|c|c|c|c|}
\hline \multirow{3}{*}{ Haplotype } & \multicolumn{5}{|c|}{ Men } & \multicolumn{5}{|c|}{ Women } \\
\hline & \multicolumn{2}{|c|}{ Frequency } & \multirow{2}{*}{ OR $(95 \% \mathrm{CI})$} & \multirow{2}{*}{$P$} & \multirow{2}{*}{$P^{*}$} & \multicolumn{2}{|c|}{ Frequency } & \multirow{2}{*}{ OR $(95 \% \mathrm{CI})$} & \multirow{2}{*}{$P$} & \multirow{2}{*}{$P^{*}$} \\
\hline & $\mathrm{HT}$ & NT & & & & $\mathrm{HT}$ & NT & & & \\
\hline$\overline{\mathrm{CCA}}$ & 0.45 & 0.42 & $1.16(0.95-1.41)$ & 0.558 & 0.162 & 0.44 & 0.42 & $1.06(0.91-1.24)$ & 0.160 & 0.460 \\
\hline CTG & 0.32 & 0.32 & $0.99(0.81-1.23)$ & 0.278 & 0.972 & 0.32 & 0.33 & $0.98(0.84-1.16)$ & 0.464 & 0.867 \\
\hline TTA & 0.18 & 0.20 & $0.89(0.69-1.15)$ & 0.319 & 0.371 & 0.17 & 0.20 & $0.84(0.69-1.02)$ & 0.551 & 0.083 \\
\hline CTA & 0.04 & 0.06 & $0.69(0.45-1.07)$ & 0.793 & 0.079 & 0.06 & 0.05 & $1.33(0.93-1.89)$ & 0.068 & 0.111 \\
\hline
\end{tabular}

Haplotypes were constructed in the order of rs2235307, rs2235306, and rs3115759.

NT: normotensive subjects. HT: hypertensive subjects.

* $P$ values were adjusted for age, body mass index and diabetes duration.

and the C-C-A haplotype with hypertension was observed (Supplemental Tables 2, 3, and 4). Next, we combined the two stages with meta-analysis (Supplemental Table 5). None of the three SNPs and the four haplotypes was significantly associated with hypertension.

\section{Discussion}

The current study genotyped three tagging SNPs of APLN region in a Chinese population with and without diabetes. The pilot data showed that the minor allele $\mathrm{C}$ of rs2335306 and the C-C-A haplotype (rs223537-rs2235306-rs3115759) were associated with a higher risk of hypertension in men with normal glucose regulation. However, our second stage samples failed to replicate this finding, and the meta-analysis also exhibited a negative result.

In 1998, Tatemoto et al. [13] isolated a 36-amino acid peptide from bovine stomach extracts and named it apelin, the endogenous ligand to an orphan G-protein-coupled receptor APJ. In human beings, preproapelin mRNA is abundant in the central nervous system, placenta and in more moderate concentrations in the kidney, heart, lungs, mammary gland, and adipocytes. Since its discovery, many of the initial investigations of the apelin/APJ system, as it relates to the cardiovascular system centered on its role in blood pressure control $[12,18,19]$. In male Wistar rats, the administration of aplein could lower systolic and diastolic blood pressure, which persisted for several minutes [19, 33]. Besides, a nitric oxide-dependent arterial vasodilation effect of apelin was also observed in vivo in men [18].

It is notable that no study has investigated whether there is difference between males and females regarding the effect of apelin on blood pressure. However, Newson et al. [34] reported that the apelin receptor (APJ) had a gender-specific function in peripheral immune activation of the hypothalamic-pituitary-adrenal axis, suggesting that the possible gender-specific influence of apelin/APJ on blood pressure may be an issue to address in the future.

Recently, Li and his colleagues [22] have assessed the associations of polymorphisms within APLN with essential hypertension in a family-based study and reported a positive result. This association was next validated in a general population by Niu et al. [23]. Nonetheless, it is notable that the associated SNPs in these two studies were not consistent. For instance, T-1860C, which was found to affect the risk of hypertension in the former study, was not successfully replicated in the latter one, where rs3761581 was observed to be risk conferring. Moreover, the $P$ values for the associations were not solid enough $(P=0.018$ and $P=0.0156$, resp.). Together with our data, it is likely that common variants of APLN alone may not exert a pronounced genetic effect on blood pressure. However, the possibility of association could not be fully excluded, as our study might have insufficient power when detecting mild genetic effects of SNPs with low minor allele frequencies. For instance, the diabetic group had 
less than $50 \%$ power to detect an OR of 1.2 for rs 2335307 (Supplemental Table 6). On the other hand, it is possible that the interaction between APLN and AGTRL1, which encodes the receptor of apelin, may have a greater genetic effect on the regulation of blood pressure. Indeed, in the study by Niu et al. [23], the association with hypertension was much more robust in the haplotype analysis, which took variants of both APLN and AGTRL1 into consideration, than in the singlelocus study restricted to APLN.

The strength of the current study resides in that a total of 6693 subjects (3156 diabetic patients and 3536 nondiabetic subjects) were recruited, which we believe is the largest sample size of study to evaluate the association between APLN and hypertension to date. But there are several limitations that should be mentioned. First, we did not obtain the information on pharmacological treatments, which might have certain effect on blood pressure. Second, common variants of AGTRL1 were not genotyped. Therefore, the effect of interaction between AGTRL1 and APLN on hypertension remained unknown. Third, we did not measure the circulating apelin concentration in our sample, precluding the analysis for the association between APLN genetic variants and circulating apelin levels.

In summary, we did not find any evidence for the association of common variants on APLN with hypertension in either diabetic or non-diabetic subjects. Although a minor effect could not be excluded, our data suggest that common variants of APLN may not play a major role in the regulation of blood pressure. Further studies in different populations are needed to confirm this finding.

\section{Conflict of Interests}

There are no conflict of interests.

\section{Authors' Contribution}

R. Zhang and J. Lu contribute equally to this article.

\section{Acknowledgments}

The authors thank all the nursing and medical staff at the Shanghai Clinical Center for Diabetes for their dedication in this study. This work was supported by grants from National 973 Program of China (2011CB504001), the National Natural Scientific Foundation of China (81170735 and 81200582), the National 863 Program of China (2012AA02A509), the Shanghai Rising-Star Program (12QH1401700), the Excellent Young Medical Expert of the Shanghai (XYQ2011041), and the Program of Shanghai Municipality for Basic Research (11JC1409600), China.

\section{References}

[1] Y. M. Barri, "Hypertension and kidney disease: a deadly connection," Current Cardiology Reports, vol. 8, no. 6, pp. 411$417,2006$.

[2] A. Mosterd, R. B. D’Agostino, H. Silbershatz et al., "Trends in the prevalence of hypertension, antihypertensive therapy, and left ventricular hypertrophy from 1950 to 1989," The New England Journal of Medicine, vol. 340, no. 16, pp. 1221-1227, 1999.

[3] J. Stokes III, W. B. Kannel, P. A. Wolf, R. B. D’Agostino, and L. A. Cupples, "Blood pressure as a risk factor for cardiovascular disease. The Framingham Study-30 years of follow-up," Hypertension, vol. 13, no. 5, pp. 13-18, 1989.

[4] R. Fagard, J. Brguljan, J. Staessen et al., "Heritability of conventional an ambulatory blood pressures: a study in twins," Hypertension, vol. 26, no. 6, part 1, pp. 919-924, 1995.

[5] G. W. Somes, G. A. Harshfield, B. S. Alpert, M. M. Goble, and R. M. Schieken, "Genetic influences on ambulatory blood pressure patterns. The Medical College of Virginia Twin Study," American Journal of Hypertension, vol. 8, no. 5, part 1, pp. 474-478, 1995.

[6] P. A. Doris, "Hypertension genetics, single nucleotide polymorphisms, and the common disease: common variant hypothesis," Hypertension, vol. 39, no. 2, part 2, pp. 323-331, 2002.

[7] V. Ruppert and B. Maisch, "Genetics of human hypertension," Herz, vol. 28, no. 8, pp. 655-662, 2003.

[8] J. Boucher, B. Masri, D. Daviaud et al., "Apelin, a newly identified adipokine up-regulated by insulin and obesity," Endocrinology, vol. 146, no. 4, pp. 1764-1771, 2005.

[9] Y. Habata, R. Fujii, M. Hosoya et al., "Apelin, the natural ligand of the orphan receptor APJ, is abundantly secreted in the colostrum," Biochimica et Biophysica Acta, vol. 1452, no. 1, pp. 25-35, 1999.

[10] Y. Kawamata, Y. Habata, S. Fukusumi et al., "Molecular properties of apelin: tissue distribution and receptor binding," Biochimica et Biophysica Acta, vol. 1538, no. 2-3, pp. 162-171, 2001.

[11] A. M. O'Carroll, T. L. Selby, M. Palkovits, and S. J. Lolait, "Distribution of mRNA encoding B78/apj, the rat homologue of the human APJ receptor, and its endogenous ligand apelin in brain and peripheral tissues," Biochimica et Biophysica Acta, vol. 1492, no. 1, pp. 72-80, 2000.

[12] D. L. Lee, R. Cheng, T. Nguyen et al., "Characterization of apelin, the ligand for the APJ receptor," Journal of Neurochemistry, vol. 74, no. 1, pp. 34-41, 2000.

[13] K. Tatemoto, M. Hosoya, Y. Habata et al., "Isolation and characterization of a novel endogenous peptide ligand for the human APJ receptor," Biochemical and Biophysical Research Communications, vol. 251, no. 2, pp. 471-476, 1998.

[14] C. Dray, C. Knauf, D. Daviaud et al., "Apelin stimulates glucose utilization in normal and obese insulin-resistant mice," Cell Metabolism, vol. 8, no. 5, pp. 437-445, 2008.

[15] L. Guo, Q. Li, W. Wang et al., "Apelin inhibits insulin secretion in pancreatic $\beta$-cells by activation of PI3-kinasephosphodiesterase 3B," Endocrine Research, vol. 34, no. 4, pp. 142-154, 2009.

[16] K. Higuchi, T. Masaki, K. Gotoh et al., "Apelin, an APJ receptor ligand, regulates body adiposity and favors the messenger ribonucleic acid expression of uncoupling proteins in mice," Endocrinology, vol. 148, no. 6, pp. 2690-2697, 2007.

[17] B. F. O’Dowd, M. Heiber, A. Chan et al., "A human gene that shows identity with the gene encoding the angiotensin receptor is located on chromosome 11," Gene, vol. 136, no. 1-2, pp. 355-360, 1993.

[18] A. G. Japp, N. L. Cruden, D. A. B. Amer et al., "Vascular effects of apelin in vivo in man," Journal of the American College of Cardiology, vol. 52, no. 11, pp. 908-913, 2008. 
[19] K. Tatemoto, K. Takayama, M. X. Zou et al., "The novel peptide apelin lowers blood pressure via a nitric oxidedependent mechanism," Regulatory Peptides, vol. 99, no. 2-3, pp. 87-92, 2001.

[20] M. Przewlocka-Kosmala, T. Kotwica, A. Mysiak, and W. Kosmala, "Reduced circulating apelin in essential hypertension and its association with cardiac dysfunction," Journal of Hypertension, vol. 29, no. 5, pp. 971-979, 2011.

[21] A. Sonmez, G. Celebi, G. Erdem et al., "Plasma apelin and ADMA levels in patients with essential hypertension," Clinical and Experimental Hypertension, vol. 32, no. 3, pp. 179-183, 2010.

[22] W. W. Li, W. Q. Niu, Y. Zhang, S. Wu, P. J. Gao, and D. L. Zhu, "Family-based analysis of apelin and AGTRL1 gene polymorphisms with hypertension in Han Chinese," Journal of Hypertension, vol. 27, no. 6, pp. 1194-1201, 2009.

[23] W. Niu, S. Wu, Y. Zhang et al., "Validation of genetic association in apelin-AGTRL1 system with hypertension in a larger Han Chinese population," Journal of Hypertension, vol. 28, no. 9, pp. 1854-1861, 2010.

[24] J. R. Sowers and M. Epstein, "Diabetes mellitus and associated hypertension, vascular disease, and nephropathy. An update," Hypertension, vol. 26, no. 6, part 1, pp. 869-879, 1995.

[25] D. Bild and S. M. Teutsch, "The control of hypertension in persons with diabetes: a public haelth approach," Public Health Reports, vol. 102, no. 5, pp. 522-529, 1987.

[26] A. S. Dye, H. Huang, J. A. Bauer, and R. P. Hoffman, "Hyperglycemia increases muscle blood flow and alters endothelial function in adolescents with type 1 diabetes," Experimental Diabetes Research, vol. 2012, Article ID 170380, 9 pages, 2012.

[27] M. T. Johnstone, S. J. Creager, K. M. Scales, J. A. Cusco, B. K. Lee, and M. A. Creager, "Impaired endothelium-dependent vasodilation in patients with insulin- dependent diabetes mellitus," Circulation, vol. 88, no. 6, pp. 2510-2516, 1993.

[28] R. M. Lindsay, R. S. Peet, G. S. Wilkie et al., "In vivo and in vitro evidence of altered nitric oxide metabolism in the spontaneously diabetic, insulin-dependent BB/Edinburgh rat," British Journal of Pharmacology, vol. 120, no. 1, pp. 1-6, 1997.

[29] W. P. Jia, C. Pang, L. Chen et al., "Epidemiological characteristics of diabetes mellitus and impaired glucose regulation in a Chinese adult population: the Shanghai Diabetes Studies, a cross-sectional 3-year follow-up study in Shanghai urban communities," Diabetologia, vol. 50, no. 2, pp. 286-292, 2007.

[30] J. C. Barrett, B. Fry, J. Maller, and M. J. Daly, "Haploview: analysis and visualization of LD and haplotype maps," Bioinformatics, vol. 21, no. 2, pp. 263-265, 2005.

[31] S. B. Gabriel, S. F. Schaffner, H. Nguyen et al., "The structure of haplotype blocks in the human genome," Science, vol. 296, no. 5576, pp. 2225-2229, 2002.

[32] Z.S. Qin, T. Niu, and J. S. Liu, "Partition-ligation-expectationmaximization algorithm for haplotype inference with singlenucleotide polymorphisms," American Journal of Human Genetics, vol. 71, no. 5, pp. 1242-1247, 2002.

[33] D. K. Lee, S. R. George, and B. F. O'Dowd, "Unravelling the roles of the apelin system: prospective therapeutic applications in heart failure and obesity," Trends in Pharmacological Sciences, vol. 27, no. 4, pp. 190-194, 2006.

[34] M. J. Newson, G. R. Pope, E. M. Roberts, S. Lolait, and A. M. O'Carroll, "Stress-dependent and gender-specific neuroregulatory roles of the apelin receptor in the hypothalamicpituitary-adrenal axis response to acute stress," Journal of Endocrinology. In press. 


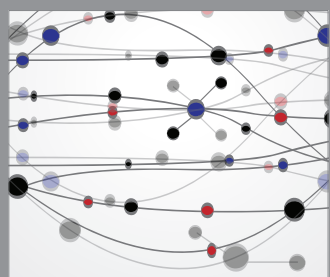

The Scientific World Journal
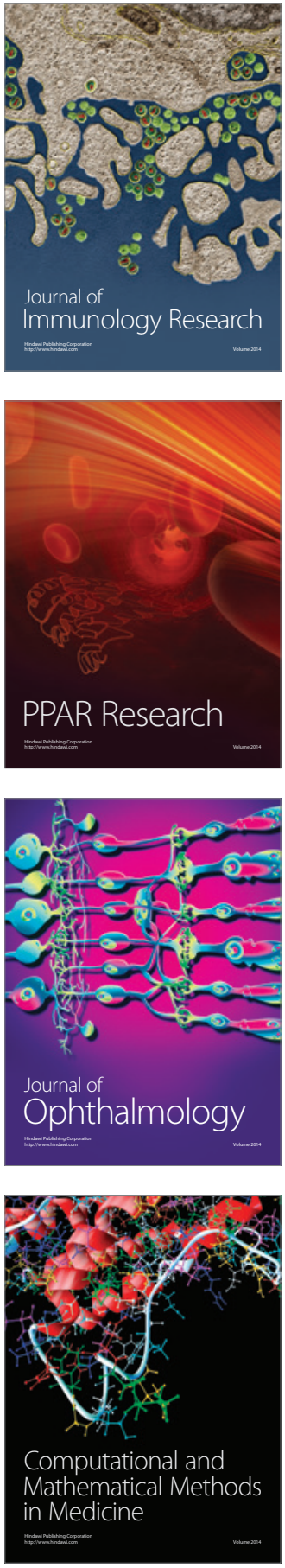

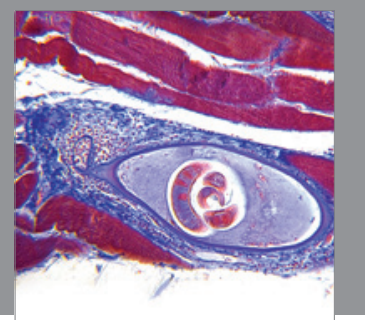

Gastroenterology

Research and Practice
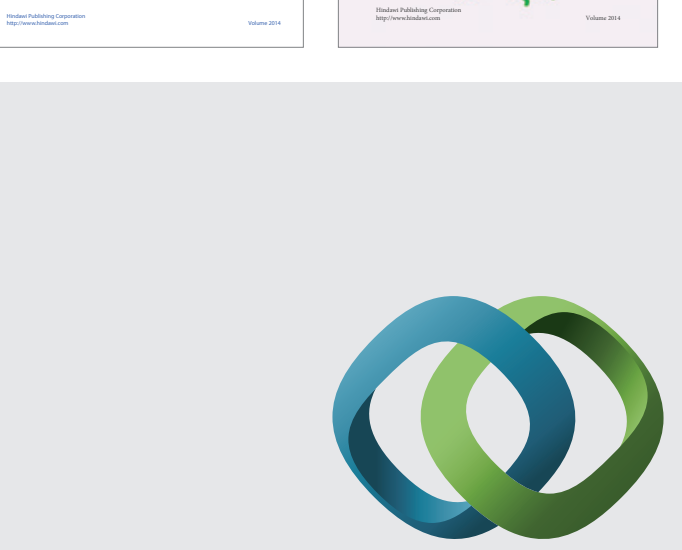

\section{Hindawi}

Submit your manuscripts at

http://www.hindawi.com
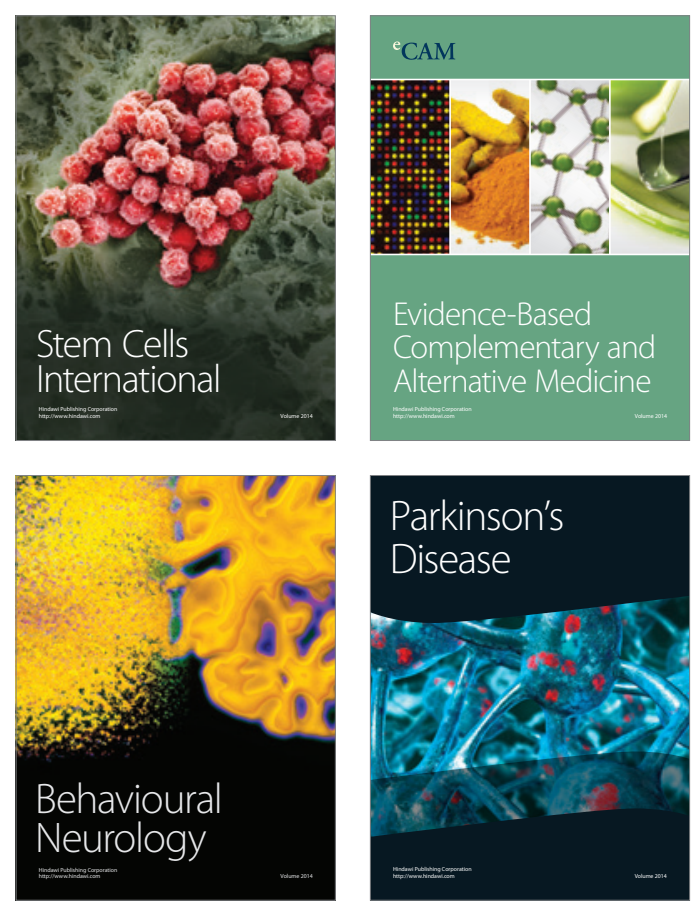

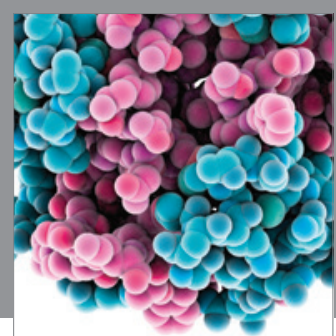

Journal of
Diabetes Research

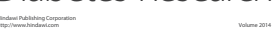

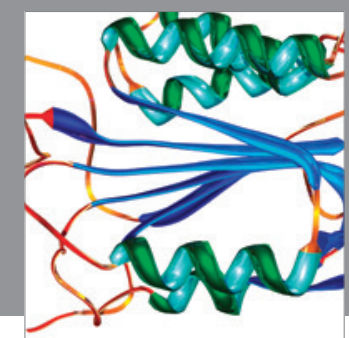

Disease Markers
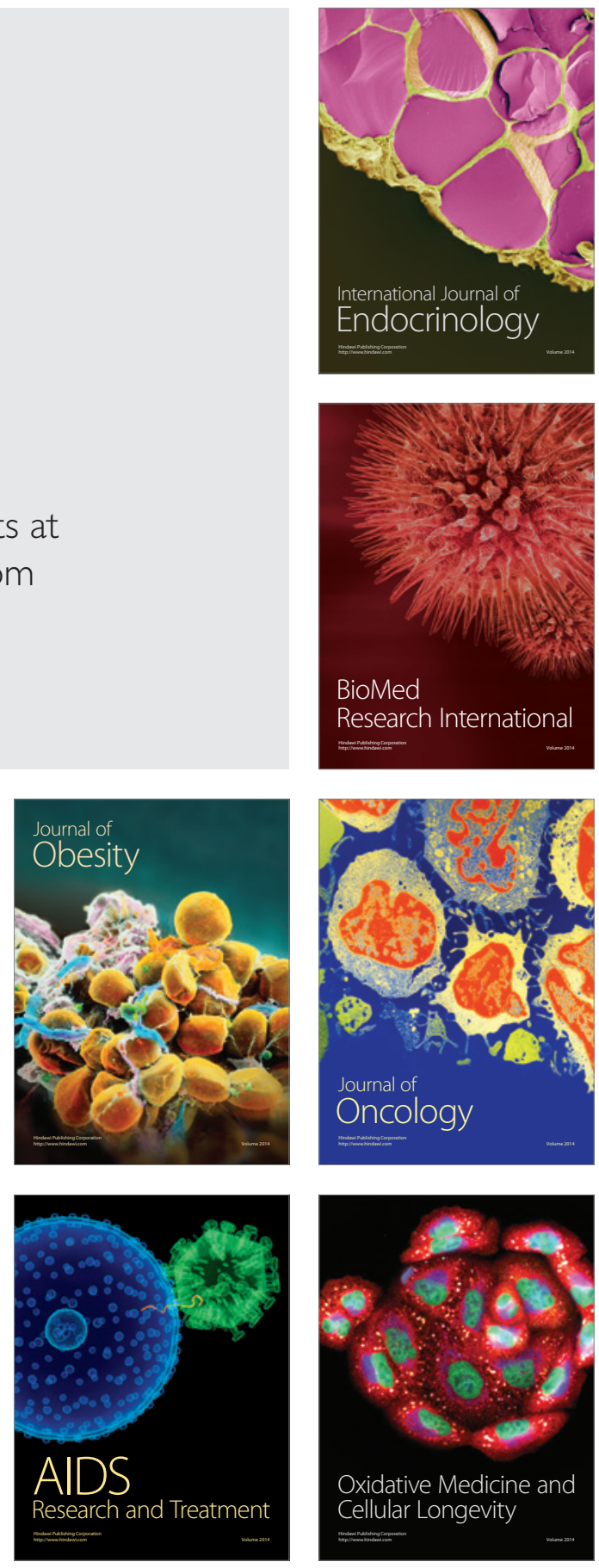